\title{
Multilateral Haptic System Stability Analysis: The Effect of Activity or Passivity of Terminations via a Series-Shunt Approach
}

\author{
Ran Tao and Mahdi Tavakoli *
}

\begin{abstract}
Haptic teleoperation and haptic interaction systems can be modeled as multi-port networks. In this context, bilateral or trilateral haptic systems stability has been analyzed in the literature by using their two-port or three-port network models. Traditionally, such stability criteria assume that operators and environments (collectively, terminations) of the multilateral haptic system are passive but otherwise arbitrary. However, recent research has shown that such an assumption can be inaccurate or too conservative as far as the human operator in a robotic system is concerned. In order not to jeopardize the haptic system stability when a termination is active or sacrifice system performance when a termination is strictly passive, we need a stability analysis approach that can take into account the degree of passivity (or lack thereof) of each termination. In response to this need, we have developed an approach based on series-shunt decomposition of the termination impedance model. Experimental validation of the theoretical stability criteria are performed involving active operators and environment for both bilateral and trilateral teleoperation.

List of Acronyms - LTI: Linear time-invariant; PEB: Position error based; RHP: Right half plane; ISP: Input strictly passive; OSP: Output strictly passive; INP: Input non-passive; ONP: Output non-passive; DNP: Disc-like non-passive; EOP: Excess of passivity; SOP: Shortage of passivity.
\end{abstract}

\section{INTRODUCTION}

Haptic teleoperation and haptic interaction systems (collectively, haptic systems) have seen increasing applications in recent years. In particular, a multilateral haptic teleoperation system can be modeled as a multi-port network representing a teleoperator (consisting of the robots, their controllers and communication channels), connected to one-port network terminations modeling the dynamics of the human operators and/or the environments. Similarly, a multilateral haptic interaction system can be modeled as a multi-port network representing a haptic virtual environment (HVE), connected to one-port network terminations modeling the dynamics of the human operators. The analysis of stability of such coupled systems can be difficult because the exact physical properties of the human operators and environments are typically unknown, uncertain or time-varying, thus making the classical approaches such as the Routh-Hurwitz criteion, inapplicable. In the presence of these uncertainties, the concept of absolute stability is often utilized. In the case of a two-port network, the absolute stability criterion ensures the coupled system's BIBO (bounded-input/bounded-output) stability for two passive but otherwise arbitrary terminations [1]. Closedform absolute stability conditions involving the two-port network's immittance parameters are given by a well-known criterion proposed by Llewellyn [2]. Thus, in traditional bilateral teleoperation, Llewellyn's criterion has been widely used for stability analysis, based on the assumption that both the human operator and the environment are linear time invariant (LTI) and passive [3, 4]. In trilateral teleoperation, our research group has developed a similar approach for absolute stability analysis based on the same passivity assumption on terminations [5].

*This research was supported by the Canada Foundation for Innovation (CFI), the Natural Sciences and Engineering Research Council (NSERC) of Canada, and the National Natural Science Foundation of China \#(61305104)

${ }^{\dagger}$ Department of Electrical and Computer Engineering, University of Alberta, Edmonton, AB T6G 2V4, Canada. E-mails:\{ran1, mahdi.tavakoli\}@ualberta.ca

IEEE Haptics Symposium 2014

23-26 February, Houston, Tx, USA

978-1-4799-3130-9/14/\$31.00 @2014 IEEE
For the human arm, although this assumption of passivity is valid when considering tasks involving a relaxed arm (or relaxed grasp) [6], it can be violated in tasks involving posture-maintenance (or rigid grasp) [7]. This could be the case in a bilateral telerehabilitation system for telepresenting a hospital-based therapist to a home-based patient in order to enable home-based rehabilitation. The reasons for considering both the therapist and the patient as active terminations are as follows. First, the therapist would be required to execute complex motor control tasks. For instance, the therapist might exert resistive forces against a patient to build muscle strength - this task bears resemblance to the rigid grasp task, which was shown in [7] to involve activity. Second, inter-muscular feedback with unequal gains has previously been linked to the arm impedance activity [8] making it possible that the patient also demonstrates an active impedance. The degree of activity can be described by the concept of shortage of passivity (SOP) defined mathematically later.

From another perspective, the assumption of having an arbitrary passive termination can lead to conservative stability conditions on the teleoperator. This happens if a termination is strictly input or output passive. For example, a mass-spring-damper system is output strictly passive in the admittance domain with an excess of passivity (EOP) equal to the system damping [9]. Excess of passivity of a termination can be used to design teleoperation systems with higher performance.

In order to derive effective stability conditions for multi-port networks involving active or strictly passive terminations, we can utilize prior knowledge concerning EOP or SOP values on the human operators or/and environments. Past work includes using Mobius transformation to map the impedance of one termination to the driving point impedance seen at the other port connected to an unknown LTI passive termination [10]. This approach, however, is limited to considering only one active or strictly passive termination for a two-port network while the other termination is simply passive. Wave variables and scattering parameters have also been used to relax Llewellyn's criterion on bounded passive terminations [11], but not for active terminations. Also, in this work, $S$-parameters are needed, but a stability condition expressed directly in the immitance (e.g. impedance) domain is more desirable, because $S$-parameters are most accurately measured for systems with higher frequency such as microwave circuits while impedance parameters can be measured accurately for mechanical systems. The approach proposed in our paper will rely solely on the impedance representation. Another approach to relax conservatism during application of Llewellyn's criterion is the series-shunt approach $[4,12]$. These papers allow taking into account the lower and upper bound on the impedance of a passive termination. However, the human operator and environment were still assumed to be passive. All of the above are limited to bilateral teleoperation systems and do not offer stability criteria for trilateral systems. In this paper, we will utilize the series-shunt approach to take into account different topologies of passive and active terminations to derive explicit stability conditions on the teleoperator, both in two-port network (bilateral) and three-port network (trilateral) cases. The same approach is applicable to $n$-port networks with larger $n$. It will also be the first time that activity or strict passivity of more than one termination and up to all terminations is allowed in the stability analysis.

The paper is organized as follows. First, passivity and activity are defined mathematically, and existing absolute stability criteria for twoport and three-port networks are reviewed in Section 2. In Section 3, the series-shunt approach is applied to two-port and three-port networks to derive stability conditions when one or multiple terminations are active or strictly passive. Although the conditions derived in Section 3 are general, they are applied to bilateral and trilateral teleoperation systems in Section 4. Closed-form conditions on controller parameters under position- 
error-based (PEB) control paradigm and experimental results are presented. Finally Section 5 contains concluding remarks and future work.

\section{Definitions and Absolute Stability Criteria for TWO-PORT AND THREE-PORT NETWORKS}

In this section, we review the definitions of passivity and activity as well as the existing stability criteria for two-port and three-port networks. Different passivity/activity topologies will be defined including input strictly passive (ISP), input non-passive (INP), output strictly passive (OSP), output non-passive (ONP), and disc-like non-passive (DNP).

\subsection{Definitions and Lemmas of passivity and activity}

Definition 1 [13] A system with input $u($.$) and output y($.$) where$ $u(t), y(t) \in \mathbb{R}^{m}$ is passive if there is a constant $\beta$ such that

$$
\int_{0}^{t} y^{T}(\tau) u(\tau) d \tau \geq \beta
$$

for all functions $u($.$) and all t \geq 0$. The constant $\beta$ is the energy stored in the system at time $t=0$. If further, there exist positive constants $\delta$ and $\varepsilon$ such that

$$
\int_{0}^{t} y^{T}(\tau) u(\tau) d \tau \geq \beta+\delta \int_{0}^{t} u^{T}(\tau) u(\tau) d \tau+\varepsilon \int_{0}^{t} y^{T}(\tau) y(\tau) d \tau
$$

for all functions $u($.$) and all t \geq 0$, the system is ISP if $\delta>0, \varepsilon=0$, and $O S P$ if $\varepsilon>0, \delta=0$. The values of $\delta$ and $\varepsilon$ are the EOP for the ISP and OSP systems, respectively.

A system is non-passive (active) if it is not passive. Based on the above definition, we can have the corresponding definition for different non-passivities:

Definition 2 In Definition 1, if $\delta<0$ then the system is INP with SOP of $\eta=-\delta$; if $\varepsilon<0$ then the system is ONP with SOP of $\mu=-\varepsilon$.

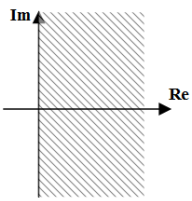

(a)

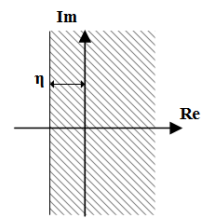

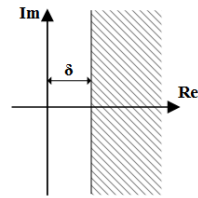

(b)

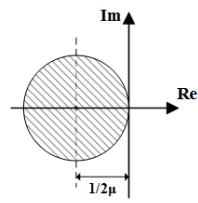

(e)

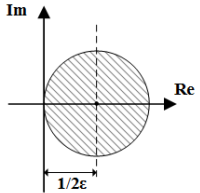

(c)

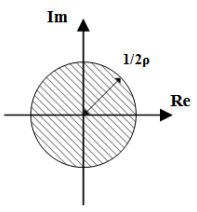

(f)
Figure 1: Nyquist diagram regions of (a) a passive system, (b) an ISP system with EOP of $\delta$, (c) an OSP system with EOP of $\varepsilon$, (d) an INP system with SOP of $\eta$, (e) an ONP system with SOP of $\mu$, (f) a DNP system with SOP of $\rho$.

When a single-input/single-output (SISO) system is represented in the frequency domain by a transfer function, the following lemmas establish the connection between different types of passivity and the region covered by the transfer function's Nyquist diagram (Fig.1):

Lemma 1 [13] A system represented by an LTI rational transfer function $G(s)$ with all poles having negative real parts is passive if and only if $\operatorname{Re}(G(j \omega)) \geq 0, \forall \omega$ (Fig. l(a)).

Lemma 2 [13] A system represented by an LTI rational transfer function $G(s)$ with all poles having negative real parts is ISP with EOP of $\delta$ if and only if $\operatorname{Re}(G(j \omega)) \geq \delta, \forall \omega($ Fig. $1(b)$ ).
Lemma 3 [13] A system represented by an LTI rational transfer function $G(s)$ with all poles having negative real parts is OSP with EOP of $\varepsilon$ if and only if $\operatorname{Re}(G(j \omega)) \geq \varepsilon|G(j \omega)|, \forall \omega$, i.e. the Nyquist diagram of $G(j \omega)$ is contained in a circle with center on the real axis at $1 / 2 \varepsilon$ and with a radius of $1 / 2 \varepsilon$ (Fig.1(c)).

By the same token, the SOP of INP and ONP systems can be related to their Nyquist diagrams in Fig. 1(d) and Fig. 1(e) respectively. Finally, we define a DNP system with its transfer function:

Definition 3 An LTI system $G(s)$ is called DNP with SOP of $\rho$ (not based on (2) but) if $|G(j \omega)| \leq 1 / 2 \rho$, i.e., the Nyquist diagram of $G(s)$ is contained in a circle centered at the origin with a radius of $1 / 2 \rho$ (Fig. $l(f))$.

The reason for considering these passivity/activity topologies is their direct physical relevance in the immittance domain. For example, the impedances of the human arm in rigid and relaxed grasping tasks have been shown to be INP and ISP, respectively [7]. Given the reciprocal relationship between impedance and admittance, it is easy to see that an INP termination in the impedance domain is ONP in the admittance domain and vice versa, while an ISP termination in the impedance domain is OSP in the admittance domain and vice versa. Thus, the admittances of the human arm in rigid and relaxed grasping tasks are ONP and OSP, respectively. Also, due to the limited co-contraction of human arm muscles, there is an upper bound on the magnitude of the arm impedance, which means the arm can always be modeled by a DNP impedance. Therefore, there is a need for considering the effect of INP, ISP, ONP, OSP and DNP immittance on stability. Note that although the rest of the paper is based on impedance parameters, the results derived here will apply to any immittance representation.

\subsection{Existing absolute stability criteria for two-port and three-port networks}

We review two absolute stability criteria in the literature that we will later use to derive stability conditions with different termination impedance topologies. Immittance-based stability criteria lend themselves well to our extension of the series-shunt approach. Therefore, first we consider an LTI two-port network which can be modeled by its impedance $(Z)$ parameters as

$$
\left[\begin{array}{l}
V_{1}(s) \\
V_{2}(s)
\end{array}\right]=\left[\begin{array}{ll}
Z_{11}(s) & Z_{12}(s) \\
Z_{21}(s) & Z_{22}(s)
\end{array}\right]\left[\begin{array}{c}
I_{1}(s) \\
I_{2}(s)
\end{array}\right]
$$

where the effort/flow pairs $\left(V_{1}, V_{2}\right)$ and $\left(I_{1}, I_{2}\right)$ denote the voltages and currents at the two terminals. A three-port networks impedance matrix relationship incorporates three pairs of effort/flow variables:

$$
\left[\begin{array}{lll}
Z_{11}(s) & Z_{12}(s) & Z_{13}(s) \\
Z_{21}(s) & Z_{22}(s) & Z_{23}(s) \\
Z_{31}(s) & Z_{32}(s) & Z_{33}(s)
\end{array}\right]
$$

Lemma 4 [1] The two-port network (3) is absolutely stable if and only if

1. $Z_{11}$ and $Z_{22}$ have no poles in the right-half plane (RHP),

2. Any poles of $Z_{11}$ and $Z_{22}$ on the imaginary axis are simple with real and positive residues, and

3. For all real positive frequencies $\omega$,

$$
\begin{aligned}
& \operatorname{Re}\left(Z_{11}\right) \geq 0 \\
& \operatorname{Re}\left(Z_{22}\right) \geq 0 \\
& 2 \operatorname{Re}\left(Z_{11}\right) \operatorname{Re}\left(Z_{22}\right)-\operatorname{Re}\left(Z_{12} Z_{21}\right)-\left|Z_{12} Z_{21}\right| \geq 0
\end{aligned}
$$

Lemma 5 [5] The three-port network with the impedance matrix $Z$ in (4) satisfying the symmetrization condition

$$
Z_{13} Z_{21} Z_{32}-Z_{12} Z_{23} Z_{31}=0
$$

is stable if and only if 


$$
\begin{aligned}
& \operatorname{Re}\left(Z_{11}\right) \geq 0 \\
& \operatorname{Re}\left(Z_{22}\right) \geq 0 \\
& \operatorname{Re}\left(Z_{33}\right) \geq 0 \\
& 2 \operatorname{Re}\left(Z_{11}\right) \operatorname{Re}\left(Z_{22}\right)-\left|Z_{12} Z_{21}\right|+\operatorname{Re}\left(Z_{12} Z_{21}\right) \geq 0 \\
& \text { and } \\
& 2 \operatorname{Re}\left(Z_{11}\right) \operatorname{Re}\left(Z_{22}\right) \operatorname{Re}\left(Z_{33}\right) \\
& -\operatorname{Re}\left(Z_{11}\right)\left(\left|Z_{23} Z_{32}\right|+\operatorname{Re}\left(Z_{23} Z_{32}\right)\right) \\
& -\operatorname{Re}\left(Z_{22}\right)\left(\left|Z_{13} Z_{31}\right|+\operatorname{Re}\left(Z_{13} Z_{31}\right)\right) \\
& -\operatorname{Re}\left(Z_{33}\right)\left(\left|Z_{12} Z_{21}\right|+\operatorname{Re}\left(Z_{12} Z_{21}\right)\right) \\
& +4 \operatorname{Re}\left(\sqrt{Z_{12} Z_{21}}\right) \operatorname{Re}\left(\sqrt{Z_{13} Z_{31}}\right) \operatorname{Re}\left(\sqrt{Z_{23} Z_{32}}\right) \geq 0
\end{aligned}
$$

\section{Main Results}

In this section we consider the two-port and three-port networks described by (3) and (4).

\subsection{Decomposition of terminations into series/parallel impedances via Mobius transformation}

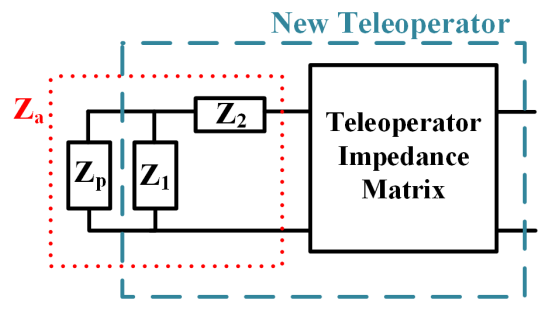

Figure 2: Decomposition of termination $Z_{a}$ into a passive impedance $Z_{p}$ in parallel connection with $Z_{1}$ and series connection with $Z_{2}$.

In order to utilize information on the topology of termination impedances so that we can derive less conservative stability conditions for overly passive terminations and valid conditions for active terminations, we propose to first decompose a given termination impedance into a passive (but arbitrary) impedance in series/shunt connection with other to-be-determined impedances. Consider a termination impedance $Z_{a}$ in Fig. 2, the impedance topology of which is known, i.e., we know whether it is passive, active (INP, ONP or DNP with a given SOP), or strictly passive (ISP or OSP with a given EOP). We decompose it into a passive impedance $Z_{p}$ in parallel connection with an impedance $Z_{1}$ and then the total in series connection with an impedance $Z_{2}$. The goal is that the overall impedance will represent the topology characterizing $Z_{a}$. Note that despite the fact that $Z_{a}$ and $Z_{p}$ are complex impedances, we have $Z_{1}, Z_{2} \in \mathbb{R}$; we will see later that they will be able to map the passive impedance $Z_{p}$ to different regions shown in Figure. 1. Now, $Z_{1}$ and $Z_{2}$ (for each termination) can be assimilated into the original two-port (or three-port) network impedance to make an augmented network, such that Lemma 4 and 5 can be applied to the new coupled system consisting of the passive $Z_{p}$ (for the corresponding termination) and the augmented network. Fig. 2 only shows the case for one termination, but the teleoperator can be augmented to incorporate $Z_{1}$ and $Z_{2}$ for as many terminations as needed. This approach for decomposing termination impedances is illustrated with INP/ISP, OSP and DNP examples in the following.

\subsubsection{Decomposition of ISP/INP terminations}

In the case of ISP and INP terminations, the complex plane regions in Fig. 1 (b) and (d) with EOP of $\delta$ and SOP of $\eta$, respectively, are obtained by a translation of the RHP along the real axis by $\delta$ or $-\eta$. Therefore $Z_{1}=\infty$, reducing the termination to a series connection of $Z_{p}$ and $Z_{2}$, with $Z_{2}$ taking the value of $\delta$ or $-\eta$.

\subsubsection{Decomposition of OSP terminations}

If $Z_{a}$ in Fig. 2 is OSP with EOP of $\varepsilon$, we can set the value of $Z_{2}$ to 0 and the value of $Z_{1}$ to $1 / \varepsilon$. The combined impedance $Z_{a}$ will be

$$
Z_{a}=\frac{Z_{p} \cdot 1 / \varepsilon}{Z_{p}+1 / \varepsilon}
$$

Which, as argued below, can characterize a given OSP impedance with EOP of $\varepsilon$. In fact, this is a Mobius transformation [14] with regard to $Z_{p}$; recall that $Z_{p}$ is any arbitrary impedance in the RHP. The transformation (8) consists of four steps:

1. Horizontal translation by $1 / \varepsilon$ of the region of the impedance $Z_{p}$, which is the RHP (Fig. 3(a) to Fig. 3(b)); i.e., $f_{1}\left(Z_{p}\right)=Z_{p}+1 / \varepsilon$

2. Inversion of the result of step 1 (Fig. 3(b) to Fig. 3(c)); i.e., $f_{2}\left(Z_{p}\right)=1 / f_{1}\left(Z_{p}\right)$

3. Scaling the result of step 2 by a factor of $-1 / \varepsilon^{2}$ (Fig. 3(c) to Fig. $3(\mathrm{~d}))$; i.e., $f_{3}\left(Z_{p}\right)=-f_{2}\left(Z_{p}\right) / \varepsilon^{2}$

4. Horizontal translation by $1 / \varepsilon$ (Fig.3(d) to Fig.3(e)) ; i.e., $f_{4}\left(Z_{p}\right)=f_{3}\left(Z_{p}\right)+1 / \varepsilon$.

It is easy to see that $f_{4}\left(Z_{p}\right)$ is the same as $Z_{a}$ in (8). Therefore, with $Z_{1}=$ $1 / \varepsilon$ and $Z_{2}=0$ we can recover the OSP termination with an EOP of $\varepsilon$.

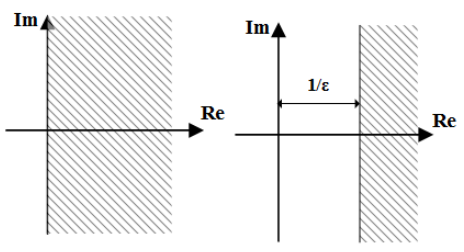

(a)

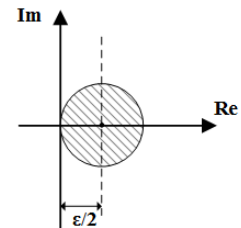

(c)

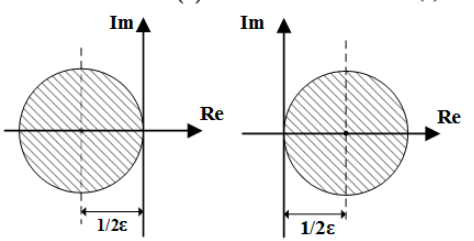

(d)

(e)
Figure 3: Step-by-step Mobius transformation of the RHP to OSP topology

\subsubsection{Decomposition of DNP terminations}

From Fig. 1(f), it can be seen that a DNP impedance with SOP of $\rho$ can be obtained by applying a horizontal translation of $-1 / 2 \rho$ to the region covered by an OSP impedance with EOP of $\rho$. Therefore, we can set the value of $Z_{1}$ to $1 / \rho$ and $Z_{2}$ to $-1 / 2 \rho$ to recover the region covered by a DNP termination in the Nyquist plane.

\subsubsection{Decomposition of ONP terminations}

Similar to the DNP termination, from Fig. 1(e) it can be seen that an ONP impedance with SOP of $\mu$ can be obtained by applying a horizontal translation of $-1 / \mu$ to the region covered by an OSP impedance with EOP of $\mu$. Therefore, we can set the value of $Z_{1}$ to $1 / \mu$ and $Z_{2}$ to $-1 / \mu$ to recover the region covered by a DNP termination in the Nyquist plane.

\subsection{Augmented teleoperator impedance with different termination combinations}

After termination impedance decomposition in Section 3.1, we can incorporate the $Z_{1}$ and $Z_{2}$ associated with each termination into the teleoperator impedance matrix to form a new teleoperator impedance matrix.

\subsubsection{Bilateral teleoperator case}

In the two-port network case, the new teleoperator impedance matrix calculation can be systematically carried out by first calculating the equivalent chain matrix for the two-port network comprised of the $Z_{1}$ and $Z_{2}$ combination at each termination. Then, the new teleoperator can be expressed in terms of its chain (ABCD) parameters by calculating 
the product of the chain matrices of the individual two-port networks as the three two-port networks are in cascade connection (Fig. 4) [1] The reason for utilizing the chain matrix instead of, say, the impedance matrix representation for each two-port network is this very ability to multiply the matrices of the cascaded networks to get the matrix for the total network. Finally, the equivalent impedance matrix of the augmented teleoperator can be obtained by performing a parameter conversion from the total chain matrix to impedance matrix.

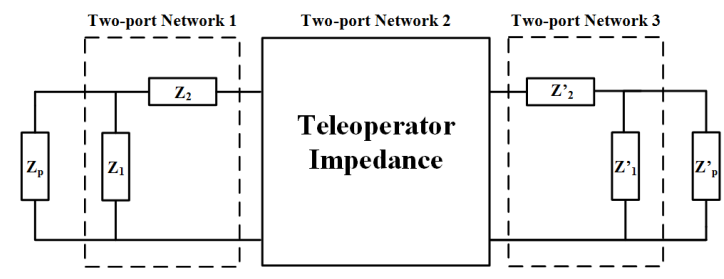

Figure 4: Cascade connection of two-port networks 1-3, consisting of the original teleoperator two-port network and two equivalent two-port networks from termination decompositions

Next, the INP, OSP and DNP terminations are considered to form different termination combinations for the two ports of the two-port network and their corresponding augmented teleoperator impedance matrices are reported in Table 1. Due to the symmetries, only six distinct cases exist: INP-INP (i.e., ports 1 and 2 are connected to INP terminations that are not necessarily the same), OSP-OSP, DNP-DNP, INP-OSP, INP-DNP, and OSP-DNP. We take termination 1's EOP/SOP to be $a$ and termination 2's EOP/SOP to be $b$. Note that it suffices to change the sign of the SOP of an INP termination in order to get the results for an ISP termination.

Stability criterion for each combination can be then derived by applying Lemma 4 to the new teleoperator impedance. An example will be studied in Section 4.

\subsubsection{Trilateral teleoperator case}

Following a similar approach, the augmented teleoperator impedance matrix can be derived for a three-port network. While a total of 35 distinct combinations exist if we consider all the termination impedance topologies mentioned so far (ISP, INP, OSP, ONP and DNP), for brevity we only report the augmented impedance matrix for one case: the three terminations are INP with SOP values of $a, b$, and $c$, respectively. The impedance matrix is

$$
\left[\begin{array}{ccc}
Z_{11}-a & Z_{12} & Z_{13} \\
Z_{21} & Z_{22}-b & Z_{23} \\
Z_{31} & Z_{32} & Z_{33}-c
\end{array}\right]
$$

Again, stability criteria can be derived for the three-port network by applying Lemma 5 to the new teleoperator impedance matrix given that we have incorporated activity or excessive passivity of the terminations into the teleoperator, leaving the terminations with only passive impedances. An example will be studied in Section 4. Note that if the symmetrization condition (6) is satisfied for the original impedance teleoperator matrix (4), it is still satisfied for the augmented impedance matrix (9).

\section{Case Studies: Application of Proposed Approach to BILATERAL AND TRILATERAL TELEOPERATION}

In this section, we apply the proposed approach for stability analysis with non-passive operator/environment to both bilateral and trilateral teleoperation systems, although the approach described above can be used for any multi-port network. As discovered in [7], human arms can exhibit INP impedance behaviors under rigid grasping conditions. Therefore, in both bilateral and trilateral cases we consider the presence of at least one INP termination. In practice, the level of activity/passivity can be determined using a similar approach in [7] where the time-domain force and velocity data are examined and definitions in Section 2 are applied. Stability criteria for each case will be derived in terms of the teleoperator impedance parameters, followed by derivation of closed-form conditions on the control gains within a given control architecture. Finally experiments are performed to validate the stability criteria. Note that although we applied the proposed approach to INP terminations only, it can be applied to any considered termination topology to derive stability conditions.

\subsection{Bilateral teleoperation under two INP terminations}

Consider a bilateral teleoperation system where the two operators acting on the master and slave robots demonstrate INP impedances of with SOP values of $a$ and $b$, respectively. As discussed in 1 this could be the case in a telerehabilitation setting. Now by applying Lemma 4 to the bilateral teleoperator matrix for the INP-INP case in Table 1, the condition set (5) is revised to

$$
\begin{aligned}
& \operatorname{Re}\left(Z_{11}\right) \geq a \\
& \operatorname{Re}\left(Z_{22}\right) \geq b \\
& 2\left(\operatorname{Re}\left(Z_{11}\right)-a\right)\left(\operatorname{Re}\left(Z_{22}\right)-b\right) \\
& -\operatorname{Re}\left(Z_{12} Z_{21}\right)-\left|Z_{12} Z_{21}\right| \geq 0
\end{aligned}
$$

Note that by setting $a$ or $b$ to zero, the stability theorem derived in [10], where only one port is terminated to an INP termination while the other port is terminated to an arbitrarily passive termination, can be recovered. However, our approach is equally applicable to bilateral teleoperation systems with one or two active terminations.

Now consider a bilateral teleoperation system under position error based (PEB) control. For details of the control architecture, readers can refer to [15]. The impedance matrix representing the system can be expressed as:

$$
\left[\begin{array}{cc}
Z_{m}+C_{m} & C_{m} \\
C_{s} & Z_{s}+C_{s}
\end{array}\right]
$$

Where, in the force-velocity domain, the master and slave robots are modeled as $Z_{m}=M_{m} s+B_{m}$ and $Z_{s}=M_{s} s+B_{s}$, and the local position controllers for the two robots are $C_{m}=K_{v m}+K_{p m} / s$ and $C_{s}=K_{v s}+K_{p s} / s$. We assume $K_{v m}, K_{v s}, K_{p m}, K_{p s} \geq 0$. Assume the operator on the master side can be characterized by an INP impedance with SOP value of $a$ while the operator on the slave side can be characterized by an INP impedance with SOP value of $b$. By applying (10) to (11), we have the following closed-form stability condition to be satisfied involving the controller gains for a given set of robot parameters:

$$
\begin{aligned}
& K_{v m}+B_{m} \geq a \\
& K_{v s}+B_{s} \geq b \\
& \left(K_{v m}+B_{m}-a\right)\left(B_{s}-b\right)+K_{v s}\left(B_{m}-a\right) \geq \\
& \frac{\left(K_{p m} K_{v s}-K_{v m} K_{p s}\right)^{2}}{4 K_{p m} K_{p s}}
\end{aligned}
$$

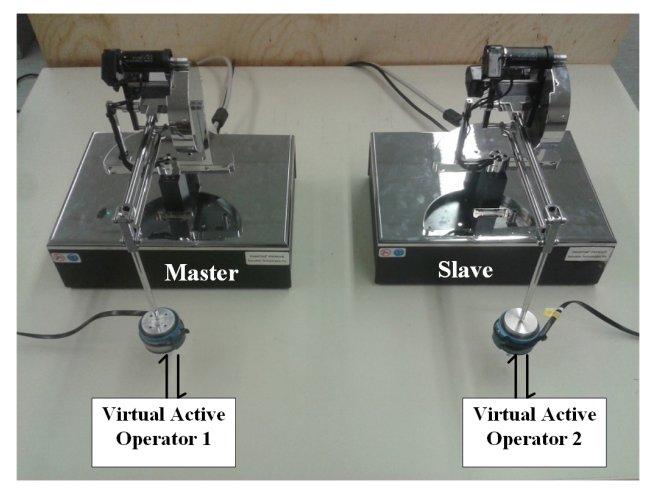

Figure 5: Bilateral teleoperation experimental setup with both the master and slave robot coupled to virtual active operators.

Next, we perform experiments to compare the stability conditions derived directly from (5) and (10); recall that (10) led to (12) and (5) would lead to the same with $a=b=0$. Bilateral teleoperation is set up as shown in Fig. 5 using a pair of 3-DOF Phantom Premium 1.5A haptic devices (Geomagic Inc., Wilmington, MA, USA). Only the first joint of each robot is teleoperated, and the other two joints are controlled 
Table 1: Augmented teleoperator impedance matrix with different termination topology combinations, where $M=Z_{22}+a\left(Z_{11} Z_{22}-Z_{12} Z_{21}\right)$, and $N=Z_{11}+b\left(Z_{11} Z_{22}-Z_{12} Z_{21}\right)$

\begin{tabular}{|c|c|c|c|c|c|c|}
\hline $\begin{array}{ll}\text { Term. } 1 & \text { Term. } 2 \\
\end{array}$ & \multicolumn{2}{|c|}{ INP with SOP of $b$} & \multicolumn{2}{|c|}{ OSP with EOP of $b$} & \multicolumn{2}{|c|}{ DNP with SOP of $b$} \\
\hline INP with SOP of $a$ & {$\left[\begin{array}{c}Z_{11}-a \\
Z_{21}\end{array}\right.$} & $\left.\begin{array}{c}Z_{12} \\
Z_{22}-b\end{array}\right]$ & {$\left[\begin{array}{c}\frac{N-a b Z_{22}-a}{1+b Z_{22}} \\
\frac{Z_{21}}{1+b Z_{22}}\end{array}\right.$} & $\left.\left.\frac{Z_{12}}{1+b Z_{22}}\right] \frac{Z_{12}}{1+b Z_{22}}\right]$ & {$\left[\begin{array}{c}\frac{N-a b Z_{22}-c}{1+b Z_{22}} \\
\frac{Z_{21}}{1+b Z_{22}}\end{array}\right.$} & $\left.\frac{Z_{12}}{\frac{-1+b Z_{22}}{2 b\left(1+b Z_{22}\right)}}\right]$ \\
\hline OSP with EOP of $a$ & & $\star$ & {$\left[\frac{N}{a N+b Z_{22}+1}\right.$} & 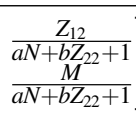 & {$\left[\begin{array}{c}\frac{N}{a N+b Z_{22}+1} \\
\frac{Z_{21}}{a N+b Z_{22}+1}\end{array}\right.$} & $\left.\begin{array}{c}\frac{Z_{12}}{a N+b Z_{22}+1} \\
\frac{a N+b Z_{22}-1-2 a Z_{11}}{2 b\left(a N+b Z_{22}+1\right)}\end{array}\right]$ \\
\hline DNP with SOP of $a$ & & $\star$ & $\star$ & & {$\left[\begin{array}{c}\frac{2 a N-b Z_{22}-1}{a\left(2 a N+b Z_{22}+1\right)} \\
\frac{2 Z_{21}}{2 a N+b Z_{22}+1}\end{array}\right.$} & $\begin{array}{c}\frac{2 Z_{12}}{2 a N+b Z_{22}+1} \\
\frac{2 a N-4 a Z_{11}+b Z_{22}-1}{2 b\left(2 a N+b Z_{22}+1\right)}\end{array}$ \\
\hline
\end{tabular}

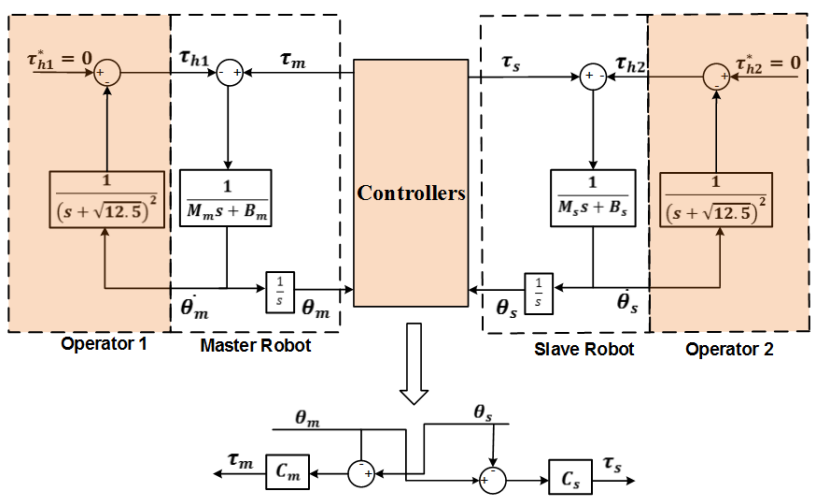

Figure 6: Schematic of the bilateral teleoperation experimental setup. The virtual systems are distinguished from physical systems by being shaded in orage.

to be locked in place by using high-gain control. The sampling time is $1 m s$. The robot dynamics are identified as mass-damper with $M_{m}=M_{s}=0.015$ and $B_{m}=B_{s}=0.01822$ in joint space [16]. The active operators are realized in the virtual environment with the transfer function $\frac{1}{(s+\sqrt{12.5})^{2}}$. The reason why we use virtual operators is that they allow us to have control over the exact SOP of each active operator, which is beneficial in validating our stability analysis approach. Plotting the Nyquist diagram of the transfer function can show an SOP of $a=b=0.01$ for the terminations. A detailed schematic of the experimental system is presented in Fig. 6, where $\tau$ and $\theta$ denote torque and angular position, respectively. Also subscripts $h 1, h 2, m, s$ denote operator 1, operator 2, the master and the slave respectively. Two sets of experiments are conducted, with the following control gains:

1. $K_{v m}=0.1, K_{p m}=20, K_{p s}=12, K_{v s}=0.1$;

2. $K_{v m}=0.1, K_{p m}=32, K_{p s}=10, K_{v s}=0.1$;

For the first set of control gains, both (5) and (10) predicted stability. As the experimental result in Fig.7(a) show, the bilateral teleoperation system is indeed stable in the presence of a persistent sinusoidal input of amplitude $0.08 \mathrm{Nm}$ and frequency $5 \mathrm{rad} / \mathrm{s}$. For the second set of controller gains, (5) predicted stability while (10) predicted instability. The experimental results in Fig.7 show that the robot positions in the teleoperation system diverge if we release the robots from two different initial conditions. Overall, the conclusion is that with active operators, Llewellyn's theorem cannot be used for controller design while the new approach can be.

\subsection{Trilateral teleoperation under one INP termination}

Consider a trilateral teleoperation system coupled to an active environment characterized by an INP impedance with a SOP value of $c$. The trilateral system considered here is a dual-user teleoperation system,

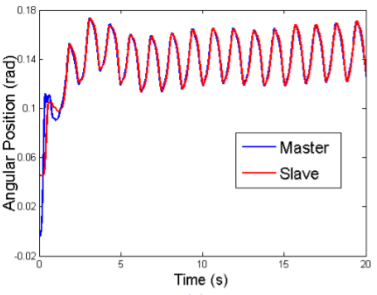

(a)

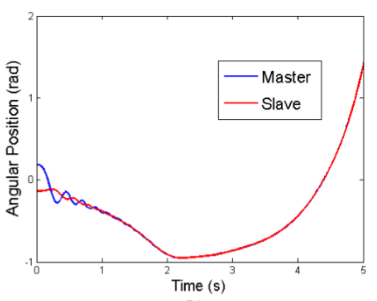

(b)
Figure 7: Bilateral master-slave joint position profiles for the first set (a) and the second set (b) of control gain.

consisting of two master robots and a remote environment. The two users collaboratively control a robot to perform a desired task. This type of trilateral systems is seeing emerging applications such as collaborative surgical training where a novice (operator 1) is trained by an experienced surgeon (operator 2) to perform a surgical task on the remote environment. In the case of beating heart surgery, for instance, the remote environment (the beating heart) is active, introducing the necessity of a stability analysis capable of dealing with non-passive environments in trilateral teleoperation. Also, motor complex tasks from either operator lead to activity of that termination for the trilateral teleoperator.

Using the three-port network model of the trilateral teleoperator and applying Lemma 5 to (9) while assuming the two users are passive $(a=b=0)$ but the environment is INP with a SOP value of $c$, conditions $(7 \mathrm{c})$ and $(7 \mathrm{e})$ are revised to

$\operatorname{Re}\left(Z_{33}\right) \geq c$,

$2 \operatorname{Re}\left(Z_{11}\right) \operatorname{Re}\left(Z_{22}\right)\left(\operatorname{Re}\left(Z_{33}\right)-c\right)$

$-\operatorname{Re}\left(Z_{11}\right)\left(\left|Z_{23} Z_{32}\right|+\operatorname{Re}\left(Z_{23} Z_{32}\right)\right)$

$-\operatorname{Re}\left(Z_{22}\right)\left(\left|Z_{13} Z_{31}\right|+\operatorname{Re}\left(Z_{13} Z_{31}\right)\right)$

$-\left(\operatorname{Re}\left(Z_{33}\right)-c\right)\left(\left|Z_{12} Z_{21}\right|+\operatorname{Re}\left(Z_{12} Z_{21}\right)\right)$

$+4 \operatorname{Re}\left(\sqrt{Z_{12} Z_{21}}\right) \operatorname{Re}\left(\sqrt{Z_{13} Z_{31}}\right) \operatorname{Re}\left(\sqrt{Z_{23} Z_{32}}\right) \geq 0$

As discussed in [17], the desired position for each robot is obtained by calculating the weighted sum of positions of the other two robots. The parameter $\alpha$ determines this weight, which can be interpreted as the relative authority of each operator over the slave robots position. By adopting the PEB control architecture in [17] and the aforementioned complementary-linear-combination (CLC) authority sharing laws, we have the following impedance matrix representing the system:

$$
\left[\begin{array}{ccc}
C_{m 1}+M_{m 1} s & -(1-\alpha) C_{m 1} & -\alpha C_{m 1} \\
-\alpha C_{m 2} & C_{m 2}+M_{m 2} s & -(1-\alpha) C_{m 2} \\
-\alpha C_{m s} & -(1-\alpha) C_{m s} & C_{m s}+M_{s} s
\end{array}\right]
$$

where the robots are modeled as $Z_{m 1}=M_{m 1} s$ and $Z_{m 2}=M_{m 2} s$ on the users side and $Z_{S}=M_{s} s$ on the environment side. Similar to the bilateral teleoperation case in Section 4.1, local position controllers are $C_{m 1}=K_{v m 1}+K_{p m 1} / s$ and $C_{m 2}=K_{v m 2}+K_{p m 2} / s$ on the users 
side and $C_{s}=K_{v s}+K_{p s} / s$ on the environment side. We assume $K_{v m 1}, K_{v m 2}, K_{v s}, K_{p m 1}, K_{p m 2}, K_{p s} \geq 0$. Note that in order to satisfy the symmetrization condition (6), $\alpha$ has to take the value of $\frac{1}{2}$. By applying the revised Lemma 5 to (14), we can get the following sufficient frequency-independent stability conditions for the trilateral teleoperation system:

$$
\begin{aligned}
& K_{v s} \geq \frac{3 c}{2} \\
& \frac{K_{v m 1}}{K_{p m 1}}=\frac{K_{v m 2}}{K_{p m 2}} \\
& \frac{5 K_{v s}-6 c-2 \sqrt{6 K_{v s}^{2}-15 K_{v s} c+9 c^{2}}}{K_{v s}} \leq \frac{K_{v s} K_{p m 2}}{K_{p s} K_{v m 2}} \\
& \leq \frac{5 K_{v s}-6 c+2 \sqrt{6 K_{v s}^{2}-15 K_{v s} c+9 c^{2}}}{K_{v s}}
\end{aligned}
$$

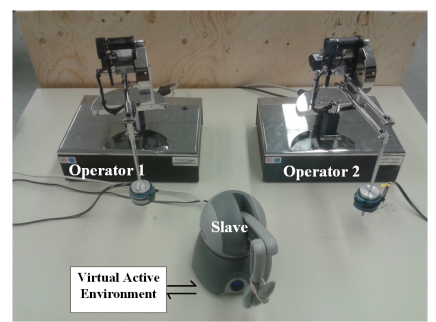

Figure 8: Trilateral teleoperation experimental setup with the slave robot interacting with virtual active environment

Finally, we put the previously-derived theoretical stability conditions to test by performing a 1-DOF trilateral teleoperation experiment. The two Phantom Premium 1.5A haptic devices used in Section 4.1 are used here as the two master robots. The slave robot at the environment side is a Phantom Omni haptic device (Geomagic Inc., Wilmington, MA, USA) (Fig. 8). Two master robots are actuated with (human operators modeled by) persistent sinusoidal inputs of amplitude $0.07 \mathrm{Nm}$, frequency $5 \mathrm{rad} / \mathrm{s}$ and phase $1.5 \mathrm{rad}$. The active environment is implemented as a virtual environment with transfer function $\frac{1}{(s+\sqrt{1.25})^{2}}$, giving an SOP value of $c=0.1$ on the remote environment. Based on (15), we chose the following stabilizing control gains (according to the theoretical stability condition): $K_{p m 1}=4, K_{v m 1}=0.1, K_{p m 2}=$ $4, K_{v m 2}=0.1, K_{p s}=8$, and $K_{v s}=0.2$. The result is shown in Fig. 9, which shows the stability of the teleoperation system.

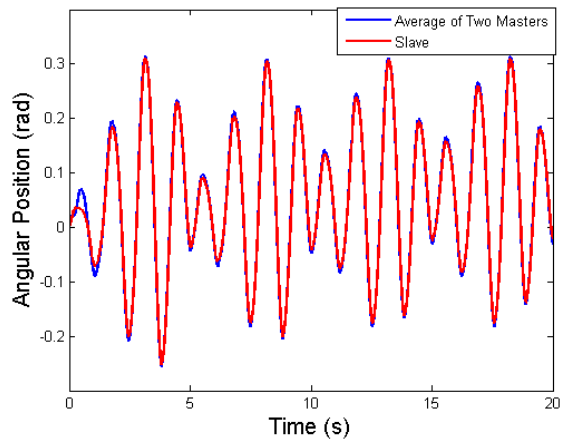

Figure 9: Robot joint positions for environment INP, dual-user PEB control.

The above demonstrates that the proposed approach can be used to provide effective controller design guidelines in the presence of active operator/environment in trilateral teleoperation.

\section{CONCLUSIONS AND FUtURE Work}

In this paper, we demonstrated the proposed series-shunt for stability analysis in the presence of strictly passive or active operators/environments for bilateral and trilateral teleoperation systems. Different from previously proposed approaches, this approach can effectively deal with strict passivity/activity in any number of terminations, easily apply to both bilateral and trilateral teleoperation, and rely solely on immittance parameters, which are relatively easy to obtain for mechanical systems. We detailed the approach for termination impedance decomposition for different impedance topologies and applied the approach to PEB bilateral and trilateral teleoperation with one or more INP terminations. Experiments were performed to validate the stability criteria and demonstrate the difference between the newly derived stability criteria and the traditional ones. In the future, the implemented active virtual operators can be replaced with robots under impedance control, so that we have a physical active system demonstrating desired active impedances. We will also investigate the implications of improved stability analysis on teleoperation transparency and thus human task performance.

\section{REFERENCES}

[1] S. Haykin, Active Network Theory. Reading, MA: Addison-Wesley, 1970.

[2] F. B. Llewellyn, "Some fundamental properties of transmission systems," Proceedings of the IRE, vol. 40, no. 3, pp. 271-283, 1952.

[3] R. Adams and B. Hannaford, "A two-port framework for the design of unconditionally stable haptic interfaces," in Intelligent Robots and Systems, 1998. Proceedings., 1998 IEEE/RSJ International Conference on, vol. 2, 1998, pp. 1254-1259 vol.2.

[4] K. Hashtrudi-Zaad and S. E. Salcudean, "Analysis of control architectures for teleoperation systems with impedance/admittance master and slave manipulators," International Journal of Robotics Research, vol. 20, no. 6, pp. $419-445,2001$.

[5] J. Li, M. Tavakoli, and Q. Huanq, "Stability analysis of trilateral haptic collaboration," in World Haptics Conference (WHC), 2013, 2013, pp. 611-616.

[6] N. Hogan, "Controlling impedance at the man/machine interface," in Proceedings of IEEE International Conference on Robotics and Automation, 1989, pp. 1626-1631 vol.3.

[7] M. Dyck, "Measuring the dynamic impedance of the human arm," Master's thesis, University of Alberta, 2013.

[8] N. Hogan, "The mechanics of multi-joint posture and movement control," Biological Cybernetics, vol. 52, no. 5, pp. 315-331, 1985. [Online]. Available: http://dx.doi.org/10.1007/BF00355754

[9] H. J. Marquez, Nonlinear Control Systems Analysis and Design. Wiley, 2003.

[10] A. Jazayeri and M. Tavakoli, "Revisiting Llewellyns absolute stability criterion for bilateral teleoperation systems under non-passive operator or environment," in Proceedings of the 2012 IEEE/RSJ International Conference on Intelligent Robots and Systems (IROS), vol. 1, Vilamoura, Portugal, 2012, pp. 70-75.

[11] A. Haddadi and K. Hashtrudi-Zaad, "Bounded-impedance absolute stability of bilateral teleoperation control systems," IEEE Transactions on Haptics, vol. 3, no. 1, pp. 15-27, 2010.

[12] R. Adams and B. Hannaford, "Control law design for haptic interfaces to virtual reality," IEEE Transactions on Control Systems Technology, vol. 10, no. 1, pp. 3-13, January 2002.

[13] R. Lozano, B. Maschke, B. Brogliato, and O. Egeland, Dissipative Systems Analysis and Control: Theory and Applications. Secaucus, NJ, USA: Springer-Verlag New York, Inc., 2007.

[14] N. Levinson and R. M. Redheffer, Complex Variables. McGraw-Hill, 1970.

[15] M. Tavakoli, A. Aziminejad, R. Patel, and M. Moallem, "High-fidelity bilateral teleoperation systems and the effect of multimodal haptics," IEEE Transactions on Systems, Man and Cybernetics - Part B, vol. 37, no. 6, pp. 1512-1528, December 2007.

[16] A. Jazayeri, M. Dyck, and M. Tavakoli, "Stability analysis of teleoperation systems under strictly passive and non-passive operator," in Proceedings of the IEEE World Haptics Conference 2013, Daejeon, Korea, 2013.

[17] B. Khademian and K. Hashtrudi-Zaad, "Shared control architectures for haptic training: Performance and coupled stability analysis," The International Journal of Robotics Research, vol. 30, pp. 1627-1642, 2011. 